\title{
Skew Reduction on a long transmission line using multiple local DLLs for high-speed imagery
}

\author{
J.-B. Kammerer, O. Maciu, I. Malass, J.-P. Le Normand and W. Uhring \\ ICube, University of Strasbourg and CNRS Strasbourg - France
}

\begin{abstract}
A transmission line induced skew reduction system for shutter signals of a pixel array has been designed using TowerJazz CIS $0.18 \mu \mathrm{m}$ technology. It is based on the use of multiple delay-locked loop in charge of close-to-close synchronization. The simulation results demonstrate the ability of the system to compensate the transmission line induced skew from $1.58 \mathrm{~ns}$ to $75 \mathrm{ps}$. The measurements made with a prototype integrating a $11.6 \mathrm{~mm}$ servo-controlled transmission line and fours delay-locked loop demonstrated a reduction of its skew down to 32ps.
\end{abstract}

Keywords—skew; imager; DLL; CMOS

\section{INTRODUCTION}

Solid-state cameras represent nowadays a viable solution for high-speed imagery applications such as fluorescence correlation spectroscopy, Raman spectroscopy, positron emission tomography as well as 3D imagery [1,2]. However, for high-speed time-resolved detection, reducing the skew of the trigger signals across large arrays remains a challenge [3]. Because of the inherent lossy nature of integrated lines, the sharp edges delivered at one of its ends bow down as the distance from the driver increases. This effect is responsible for a skew which limits the temporal resolution of the system. Matched signal trees placed on the longer side of the arrays to

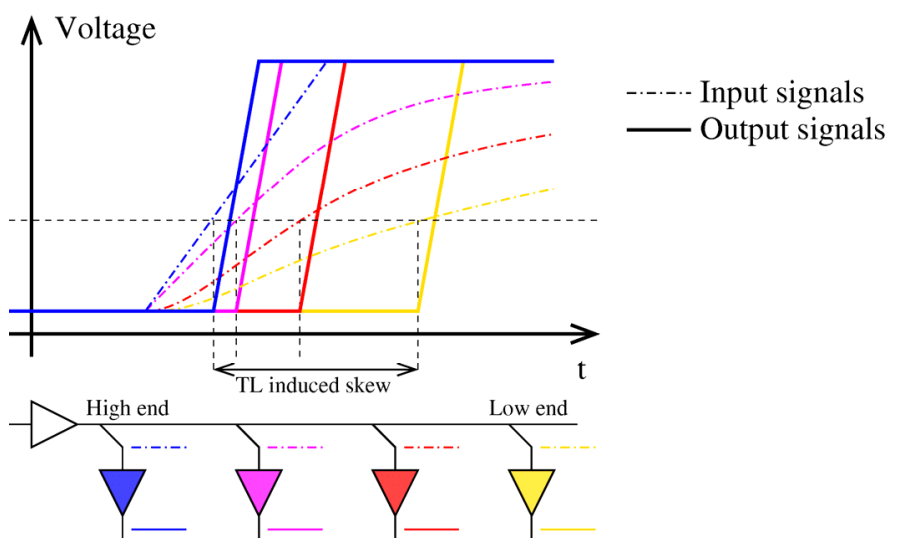

Figure 1: Shape of a rising edge seen at different positions on a long transmission line (TL). The delay between the trigger signal provided at the high end and the effective trigger signal received inside a pixel increases with the distance from the line driver. minimize the length of each transmission line are generally used to minimize this effect and skews of the order of hundreds picoseconds are reported [4,5]. However, by tuning the delay of the pixels thanks to programmable delay cells, a skew as low as $8 \mathrm{ps}$ as been demonstrated, but after a complex calibration procedure [6]. Other simpler approaches such as the use closeto-close synchronization of small and very simple ring oscillators [7] or the tuning of the threshold voltages of logic gates [8] were recently proposed and demonstrated encouraging results.

\section{PROPOSED ARCHITECTURE}

\section{A. Principle}

Because of the inherent lossy nature of integrated lines, the sharp edges delivered at the input of a transmission line (TL) bow down as the distance from the driver increases. This effect depicted in figure 1 is responsible for a skew which limits the temporal resolution of the system [8].

To improve the synchronization of the pixels triggering signal of a high-speed imager, we chose to accommodate ourselves the integrity loss of the signal along a TL. We thus searched for a mean to obtain the same response delay for all the pixels while they do not see the same signal shape. As shown in figure 2 , this feature can be obtained by adjusting the threshold voltages of the trigger inputs of each pixel according to their position along the TL: in the case of a rising edge, by setting a high threshold voltage for the first pixel and

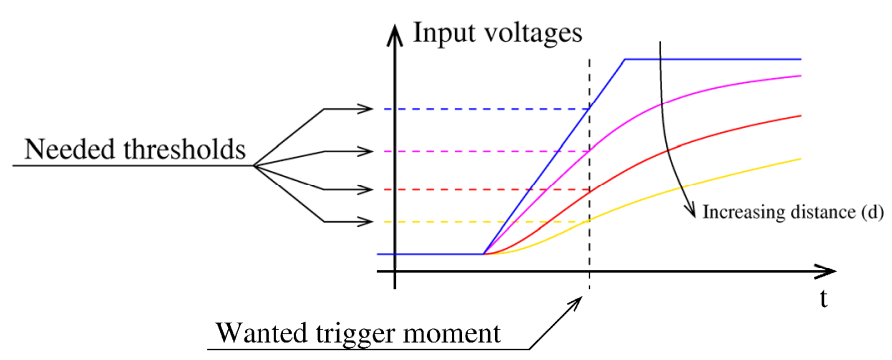

Figure 2: The synchronization of pixels placed at different distances from the line driver can be achieved by adjusting their input threshold voltages. In the case of a rising edge, the threshold voltage must be decreased as the distance increases. 


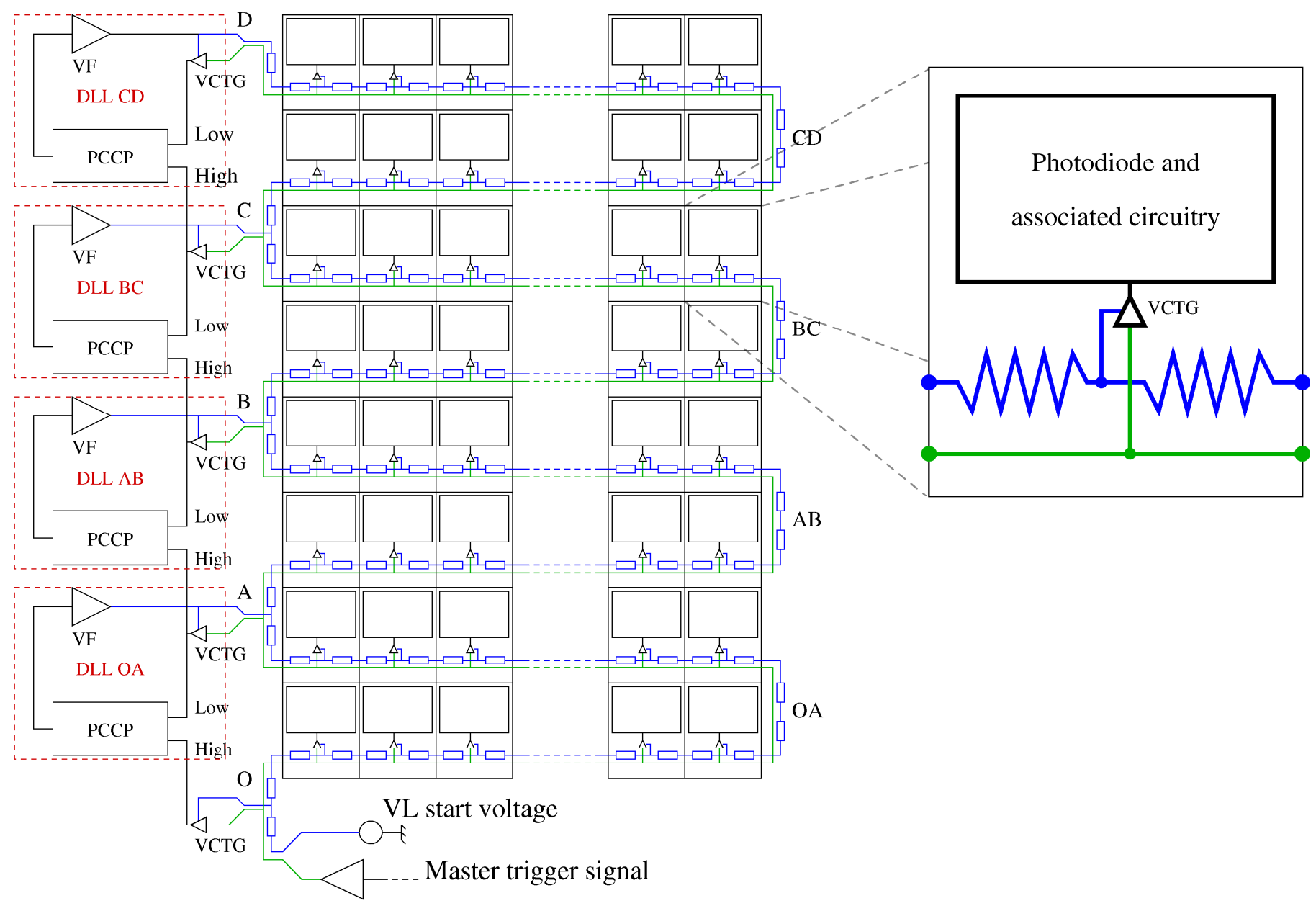

Figure 3: Architecture of the servo-controlled transmission line. The local differences of delays at the output of VCTGs connected to the transmission line (TL in green) are minimized thanks to four phase comparators and charge pumps (PCCPs). The output control voltages are applied on the corresponding low sides access points of the voltage ladder (VL in blue) thanks to voltage followers (VFs).

progressively decreasing it for the following pixels as the distance between them and the line driver increases should theoretically give rise to a perfect synchronization of the effective triggering of the pixels. It would be the opposite in the case of a falling edge, and thus adjusting the thresholds to reduce the skew on the rising edges will increase the skew on the falling edges and vice versa. As the pixels only react on rising edges, this drawback has no effect on the behavior of our imager.

We inserted in the trigger receiving circuits of the pixel a gate whose input threshold level can be controlled by an external voltage (VCTG). It consists in two CMOS inverters, the first one being degenerated thanks to two transistors (M2,M3) in series with the sources of the transistors (M0,M1) inverter (figure 4). The gates of these additional transistors are connected to the threshold control input. These transistors work in linear region and thus behave as two complementary adjustable resistors: the channel resistance of M3 is decreased (resp. increased) while the channel resistance of M2 is increased (resp. decreased) when VCtrl is increased (resp. decreased). This simple structure allows to electrically adjust the threshold control voltage of each pixel independently. However, adjusting the threshold voltage of each individual pixel independently would require one wired per pixel which is not feasible in a pixel array. To circumvent this difficulty, we chose to make use of a voltage ladder (VL) made of resistors in series to provide the individual control voltages. The total resistance of the VL is equal to $2 \mathrm{Mohm}$. The additional power consumption induced by approach is thus negligible (lower than 500nA, depending on the actual applied voltages difference). This approach allows to obtain a linear distribution of the threshold control voltage along the VL. However, because neither the needed threshold level versus distance from the line driver, nor the threshold versus control voltage characteristics are linear functions, only biasing both ends of the VL is not sufficient to accurately adjust the threshold voltages. We thus made use of few intermediate access points to adjust the voltages inside the resistors chain. With these intermediate access points, one can generate a piecewise linear voltages distribution to better fit the ideal distribution that would lead to a perfect cancellation of the TL induced skew. 


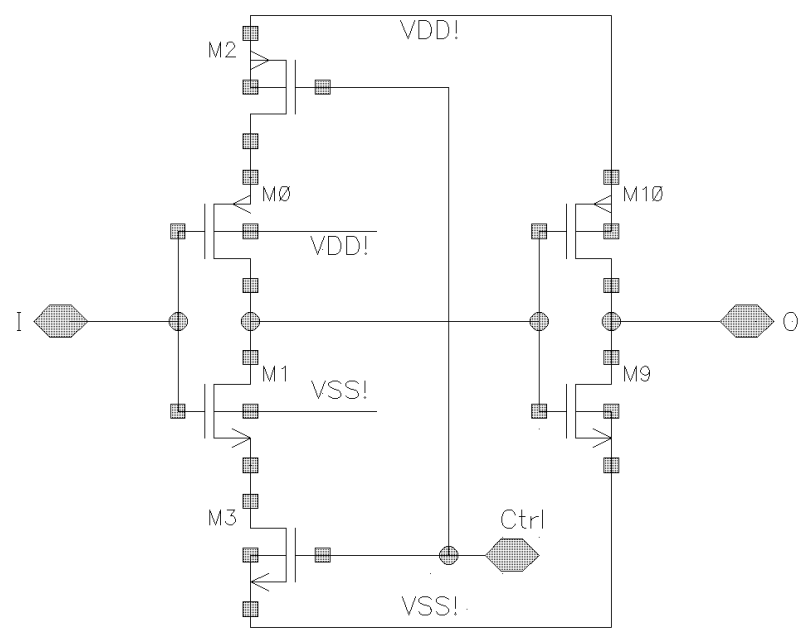

Figure 4: Schematic of the Voltage Controlled Threshold Gate

The elementary pixel layout includes a piece of TL and a piece of VL made of two resistors in series. This layout allows to automatically generate horizontal TLs and VLs inside the array when placing pixels side by side. The pixel array is then made by stacking horizontal lines of pixels which include horizontal TLs and VLs. Finally, these horizontal TLs and VLs are chained thanks to U-shaped connectors placed on the side of the array to build a single serpentine like TL and a single VL of the same shape (figure 3 ). However, the addition of the two resistors and the VCTG increases the area of the pixel of $40 \%$. The fill factor of the pixel is thus reduced in the same proportions.

\section{B. Advantages of the serpentine shape}

This approach mays seem inadequate as it maximizes the length of the TL, but the serpentine shape has the advantage of carrying back the trigger signal on each side of the array every two rows. It thus becomes possible to easily monitor the

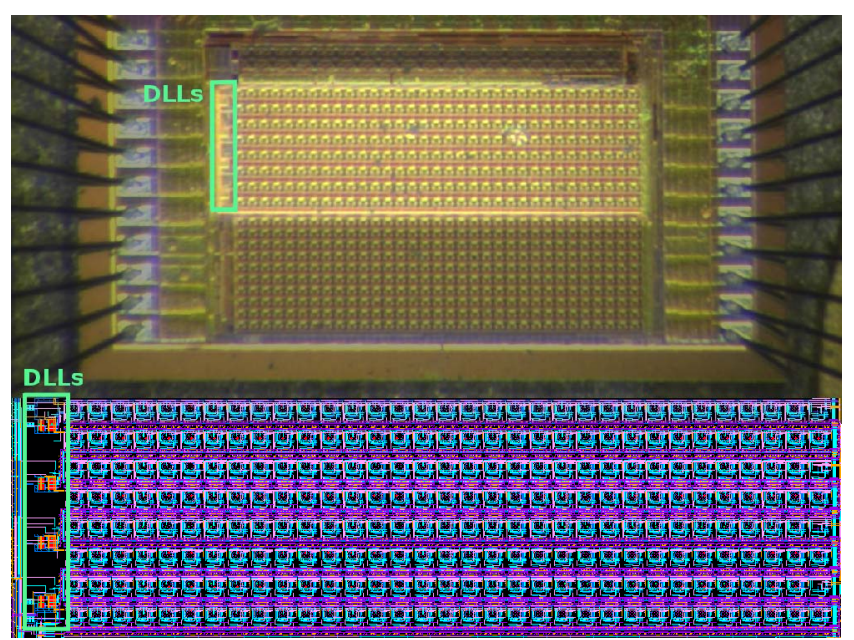

Figure 5: Layout and microphotograph (highlighted part only) of the $33 \times 8$ pixels array $\left(1400 \times 430 \mu^{2}\right)$ synchronized by 4 DLLs (in green).

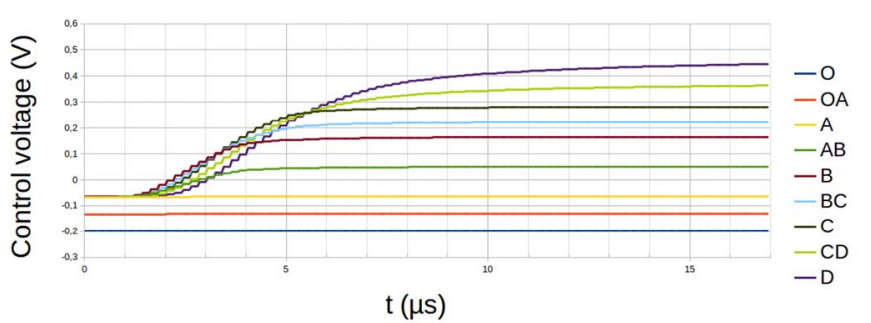

Figure 6: Simulated control voltages along the voltage ladder during the locking process. The voltage of the first node $(O)$ is fixed by a DC voltage source (see figure 3).

effective trigger signals obtained along the line at different distances from its driver by placing dedicated circuitries between adjacent U-shaped connectors. The serpentine shape also gives access to intermediate nodes of the VL allowing to adjust their potentials to accurately synchronize the effective triggers after each back and forth. For this purpose, we made use of delay-locked loops (DLLs) between each pair of adjacent U-shaped connectors of the left side of the matrix to monitor the two signals obtained at the output of VCTGs identical to the ones used in the pixels and connected to these adjacent connectors (figure 4)

\section{Close-to-close synchronization}

The difference of delays of the rising edges obtained at the outputs of the side VCTGs used to observe the effective trigger signals along the TL is measured thanks to a phase comparator. This phase comparator controls a charge pump that acts as an error integrator. Thanks to two matched switched current sources, a charge proportional to the effective delays difference is injected in an integrating capacitor. The output voltage is thus increased when the edge of the low-side VCTG (see figure 4) comes after the edge of the high-side VCTG, and it is decreased if the edge of the low-side comes before the one of the high-side. This voltage is finally applied by a voltage follower (VF) on the low-side of the corresponding part of the VL leading to locally servo-controlled voltages inside the ladder that synchronize the effective trigger signal obtained on the left side of the array. Thanks to this close-to-close servocontrol technique, the voltages distribution obtained along the VL should follow a piecewise linear approximation of the ideal voltage distribution, the starting potential of the VL being imposed by a constant voltage source to set the threshold of the first pixel of the line to its highest possible value.

\section{EXPERIMENTAL RESULTS}

A prototype of this system has been developed using TowerJazz CIS $0.18 \mu \mathrm{m}$ technology. It consists in a $33 \times 8$ pixels $\left(1400 \times 430 \mu \mathrm{m}^{2}\right)$ array with four DLLs placed on its left side to locally adjust the threshold control voltages (figure 3 and 5). The total length of the servo-controlled TL of the designed prototype is $11.6 \mathrm{~mm}$. Its behavior has been first simulated using Spectre under the Cadence's environment. The results show how the control voltages are close-to-close adjusted (figure 6) so that the rising edges obtained at the output of the 


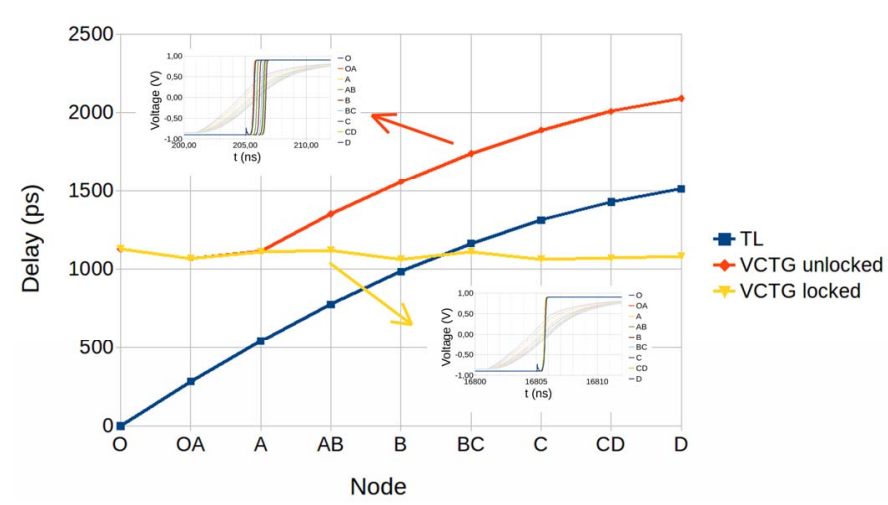

Figure 7: Simulated delays along the transmission line at the inputs (blue) and at the outputs of the VCTGs before (red) and after (yellow) the locking process. The overlaying figures show the corresponding input (light curves) and output edges of the monitored VCTGS. The delays are relative to the input edge of the first VCTG on node $O$ (see figure 3).

VCTGs placed on the side connectors get progressively closer to the rising edge obtained at the output of the first VCTG on node $\mathrm{O}$ (figure 7). This simulation demonstrates a dramatic decrease of the skew from $1.51 \mathrm{~ns}$ to only $68 \mathrm{ps}$ after 90 periods (figures 6 and 7) on a $11.6 \mathrm{~mm}$ long TL. On the actual prototype, the most right pixel of each odd line were replaced by some so called "digital pixels". They are standard pixels where the photodiode is removed to insert circuitries allowing to monitor the signals received inside the pixels. We were thus able to monitor the effective trigger signals of the pixels placed on the right side of the array (nodes $\mathrm{OA}, \mathrm{AB}, \mathrm{BC}$ and $\mathrm{CD}$, see figure 3 ), the local synchronizations being computed on the left side (nodes A, B, C and D) of the array. To obtain an estimation of the intrinsic skew of the TL, we drove the master trigger input with a low frequency pulse signal to prevent the DLLs from adjusting the voltages in the VL. At too low frequency, the charges injected in the integrating capacitor of the charge pumps are lost because of the leakage currents before the next cycle. In these conditions, the control voltages of the input gates of the pixels are all approximately identical and a skew of 572ps has been reported. In a second experiment, we drove the trigger input with a $5 \mathrm{MHz}$ pulse signal. At this frequency, the charge pumps work properly and the intermediate control voltages are servo-controlled. The voltage at the first end of the VL was fixed to a low value to set the threshold level of the first pixel at a high level. As expected, the intermediate voltages inside the VL were automatically adjusted to reduce the skew and a residual difference of delays of $32 \mathrm{ps}$ between the observable nodes was reported (figure 8)

\section{CONCLUSION}

A system allowing to automatically compensate the skew on a long transmission line intended to deliver the shutter signal over a pixels array has been developed. It relies on the use of a

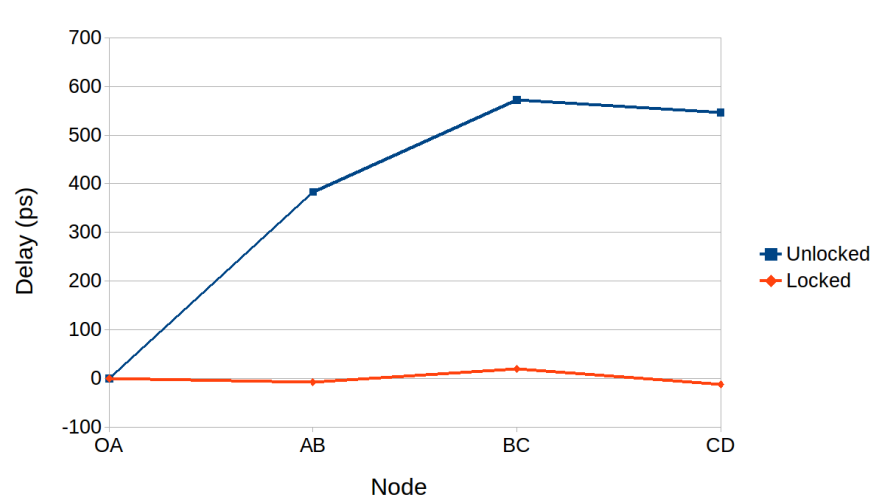

Figure 8: Measured delays through the "digital pixels" placed on the right side of the array, the DLLs being placed on the left side. All the delays are relative to the first "digital pixel" (node $O A$ ).

single transmission line with the shape of a serpentine coupled to input gates with voltage-controlled threshold, the control voltages being provided by a voltage ladder of the same shape. This particular shape allows to monitor the signal along the line every two rows to locally adjust the control voltages thanks to four DLLs. A residual skew of 32 ps on a $11.6 \mathrm{~mm}$ long lossy line was reported.

\section{ACKNOWLEDGMENT}

We thank the Agence Nationale de la Recherche for financial support under grant ANR-14-CE26-0024-01

\section{REFERENCES}

[1] D. Bronzi et al, “100,000 Frames/s 64×32 Single-Photon Detector Array for 2-D Imaging and 3-D Ranging”, IEEE J Quant Elect, Vol. 20, n6, DOI: 10.1109/JSTQE.2014.2341562, 2014

[2] F. Villa et al, "CMOS Imager With 1024 SPADs and TDCs for SinglePhoton Timing and 3-D Time-of-Flight, "IEEE Journal of Selected Topics in Quantum Electronics, Vol. 20, 3804810, DOI: 10.1109/JSTQE.2014.2342197, 2014

[3] A. Matwyschuk et al, "A real time 3D video CMOS sensor with time gated photon counting," IEEE NEWCAS, DOI: 10.1109/NEWCAS.2017.8010104, 2017

[4] Y. Maruyama et al, "A $1024 \times 8$ 700ps Time-Gated SPAD Line Sensor for Raman Spectrometry and LIBS in Space and Rover-Based Planetary Exploration,” IEEE ISSCC, DOI: 10.1109/ISSCC.2013.6487659, 2013

[5] S. Burri et al, "Architecture and applications of a high resolution gated SPAD image sensor," Optics Express, DOI:10.1364/OE.22.017573, 2014

[6] K. Yasutomi et al, "A $0.3 \mathrm{~mm}$ Resolution Time-of-Flight CMOS Range Imager with Column-Gating Clock-Skew Calibration," IEEE ISSCC, DOI: 10.1109/ISSCC.2014.6757369, 2014

[7] A.R. Ximenes, P. Padmanabhan, E. Charbon, "Mutually Coupled Ring Oscillators for Large Array Time-of-Flight," Imagers IISW 2017, Hiroshima, 2017

[8] O. Maciu, W. Uhring, J-B. Kammerer, J-P. Le Normand, N. Dumas, F. Dadouche, L. Hébrard, "Sub-Nanosecond Gated Photon Counting for High Spatial Resolution CMOS Imagers," IEEE NEWCAS 2016, Vancouver, Canada, pages 1-4, IEEE, November 2016, DOI: 10.1109/NEWCAS.2016.7604816 